\title{
AN INTERMEDIARY PERIODIC ORBIT FOR HYPERION
}

\author{
I. STELLMACHER \\ Bureau des longitudes, Université Lille I
}

\begin{abstract}
We have seen (Stellmacher, 1996) that the long-period terms for Hyperion's motion are very well represented by a second kind and second genius periodic orbit, after Poincaré's classification.

We have shown how to construct such an orbit with, as only data, the observed periods, which characterise the resonance of the Titan-Hyperion couple, and the Titan's motion which is an elliptical one. The physical quantities as the masses and the $J_{2}$ term of Saturn's flattening are given. We will present the results that we obtained, and compare them with those that other authors obtained by fitting the series to the observations.
\end{abstract}

\section{Description of the method}

Let $T_{H}, T^{\prime}, T_{\omega}$ be the observed periods for Hyperion's and Titan's mean anomalies and for the longitude of Hyperion's pericenter and $n_{H}, n^{\prime}, n_{\omega}$ the corresponding frequencies. We have $4 n_{H}-3 n^{\prime}+3 n_{\omega}=0,-n_{H} / n_{\omega} \equiv N_{H}=332$ and $-3 n / n_{\omega} \equiv N_{T}=1325$. We put : $T_{H}=k \bar{T} / N_{H}, T^{\prime}=3 k \bar{T} / N_{H}$ and $T_{\omega}=k \bar{T}$ with $k \in N$ and $\bar{n}=2 \pi / \widetilde{T}$. We have then $4 N_{H}-N_{T}-3=0$ and $N_{H}, N_{T}$ and $k$ characterise the resonance.

If $n^{\prime}$ is known, all the other frequencies can be calculated and must fit to the observations. With $n^{\prime}=22.57697385^{\circ} / j$ (see Garcia, 1972) then $n_{\omega}=$ $-3 n^{\prime} / N_{T}=-0.05111755^{\circ} / j, n_{H}=3 n^{\prime} N_{H} / N_{T}=16.971068645^{\circ} / j$ and if $k=11, \bar{n}=0.5622944^{\circ} / j$. These calculated data are very close to the observed ones.

In order to determine the semi-major axis, we define: $n_{H}=n_{0}\left(1+\varepsilon \gamma_{H}\right), n_{0}$ the mean motion corresponding to the observed semi major axis of Hyperion $a_{0 H}$, $a_{H}$ the semi major axis of a keplerian orbit corresponding to $n_{H}, a^{\prime}$ the semi major axis of Titan's orbit, $m^{\prime}, M$ the masses of Titan and Saturn, $\varepsilon=m^{\prime} / M, n^{\prime 2} a^{\prime 3}=$ $n_{H}^{2} a_{H}^{3}=n_{0}^{2} a_{0 H}^{3}=\mu, n_{H}^{2} a_{0 H}^{3}=\mu\left(1+\varepsilon \gamma_{H}\right)^{2} \neq \mu$ and $\rho_{0 H}=a^{\prime} / a_{0 H}, \rho_{H}=$ $a^{\prime} / a_{H}$. Hyperion's perturbed motion does not verify the third Keplerian law and the semi major axis $a_{0 H}$ of the real orbit will be determinated after having calculated the quantity $\varepsilon \gamma_{H}$.

Let $R_{1}=G m^{\prime} / \Delta=\left(G m^{\prime} / a_{0 H}\right) .\left(a_{0 H} / \Delta\right)$ (with $\Delta=$ mutual distance HyperionTitan), be the principal part of the disturbing function. Let $r, v, 1, \omega$ be the radius vector, the true and mean anomalies, and the longitude of the pericenter of Hyperion (the primed quantities are those for Titan), and put $\rho=r / r^{\prime} ; \theta=\omega-\omega^{\prime}$ and $\psi=4 \ell-3 \ell^{\prime}+3 \theta$.

As we explained in (Stellmacher, 1996), the disturbing function is developped by taking Saturn's flattening and Titan's action upon Hyperion into account. As far as Titan's action upon Hyperion is concerned, we only consider the $n \psi \pm m \theta(n \leq 12$ if $m=0 ; n \leq 9$ if $m=1$ or 2 ).

\section{Results}

Let $\tau=\bar{n}\left(t-t_{0}\right), \xi=n_{\omega} t+\omega_{0}-\omega_{0}^{\prime}, \rho_{0 H}=a^{\prime} / a_{0 H}$ and $a, e, \omega, \lambda$ the semi major axis, the eccentricity, the longitude of the pericenter, and the longitude. 
In the first case (I) we have calculated $\rho_{0 H}, e_{0}$ with only the $n \psi$ arguments, $\beta_{0}$ and $\bar{n}$ are calculated by replacing $\theta$ by $\xi$ for the $n \psi \pm \theta$ and $n \psi \pm 2 \theta$ terms. In the second case (II), we have calculated $\rho_{0}, e_{0} \beta_{0}$ and $\bar{n}$ with $n \psi, n \psi \pm \theta ; n \psi \pm 2 \theta$ arguments with $\theta=\xi-\beta_{0} \sin \xi$. The quantities in brackets (III) are the minimal and maximal values determined by different authors (see references).

(I) $\rho_{0 H}=0.824846$

$$
\begin{aligned}
& a=a_{0}(1-0.00327 \cos \tau) \\
& e=0.10440-0.00395 \cos \tau+0.0245 \cos \xi-0.00183 \cos 2 \xi \\
& \omega=n_{\omega} t+\omega_{0}-13^{0} 47 \sin \xi+1^{0} 64 \sin 2 \xi-0^{0} 49 \sin \tau-0^{0} 32 \sin (\xi-\tau)-0^{0} 27 \sin (\xi+\tau) \\
& \lambda=\left(n_{\omega}+n_{H}\right) t+\lambda 0+9^{\circ} 05 \sin \tau+0^{0} 25 \sin (\xi-\tau)+0^{0} 20 \sin (\xi+\tau)
\end{aligned}
$$

(II) $\rho_{0 H}=0.824825$

$$
\begin{aligned}
a & =a_{0}(1-0.00332 \cos \tau) \\
e & =0.10384-0.00403 \cos \tau+0.0260 \cos \xi-0.00160 \cos 2 \xi \\
\omega & =n_{\omega} t+\omega_{0}-13^{0} 09 \sin \xi+1^{0} 50 \sin 2 \xi-0^{0} 50 \sin \tau-0^{0} 31 \sin (\xi-\tau)-0^{0} 26 \sin (\xi+\tau) \\
\lambda & =\left(n_{\omega}+n_{H}\right) t+\lambda 0+9^{0} 18 \sin \tau+0^{0} 25 \sin (\xi-\tau)+0^{0} 21 \sin (\xi+\tau) \\
\text { (III) } \rho_{0} H=0.824942 & \\
a & =a_{0}(1-[0.00323,0.00354] \cos \tau) \\
e & =[0.10346,0.10473]-[0.00389,0.00410] \cos \tau+[0.0230,0.0245] \cos \xi-[0.00110,0,0014] \cos 2 \xi \\
\omega & =n_{\omega} t+\omega_{0}-\left\{12^{0} 87,13^{0} 58\right] \sin \xi+\left[1^{0} 56,2^{0} 16\right] \sin 2 \xi-\left[0^{\circ} 43,0^{0} 45\right] \sin \tau-\left[0^{0} 354,0^{0} 360\right] \sin (\xi-\tau) \\
& -\left[0^{0} 263,0^{0} 265\right] \sin (\xi+\tau) \\
\lambda & =\left(n_{\omega}+n_{H}\right) t+\lambda 0+\left[9^{\circ} 089,3^{\circ} 142\right] \sin \tau+\left[0^{0} 210,0^{0} 220\right] \sin (\xi-\tau)+\left[0^{0} 190,0^{0} 228\right] \sin (\xi+\tau)
\end{aligned}
$$

\section{References}

Blitzer, L.: 1977, Cel. Mech. \& Dyn. Astro., 16, 87-95.

Blitzer, L., Anderson, J.P.: 1981, Cel. Mech. \& Dyn. Astro., 25, 65-78.

Brouwer, D. and Clemence, G. M. :1961, Celestial Mechanics, Academic Press, New York.

Dourneau, G.: 1993, Astron. Astrophys., 267, 292-299.

Duriez, L.:1988, Astron. Astrophys., 194, 309-318.

Ferraz Mello, S.: 1987, Astron. Astrophys., 183, 397-402.

Ferraz Mello, S., Sato, M.,:1989, Astron. Astrophys., 225, 541,47.

Garcia, H.A.: 1972, A.J., 77, . 684.

Haag, J.: 1948, J. Ann. Sci. de l'E.N.S., 64 , 299.

Hagihara, Y.: 1971, Celestial Mechanics, 2 part 1., M.I.T. Press.

Message, P. J.: 1987-1988, Cel.Mech. \& Dyn. Astro., 43, 119-125.

Message, P. J.: 1988-1989, Cel. Mech. \& Dyn. Astro., 45, 45-53.

Message, P. J.: 1993, Cel.Mech.\& Dyn. Astro., 56, 277-284.

Poincaré, H.: 1987, Méthodes nouvelles de la mécanique céleste, réimpression par A. Blanchard de l'édition Gauthiers-Villars, Tome 1, p. 79, Tome 3, p. 201.

Rosean, M.: 1966, Vibrations non linéaires et théorie de la stabilité, Springer Tracts in Natural philosophy, Vol. 8, Berlin, Heidelberg, New-York.

Stellmacher, I.: 1996, IV International Workshop on Positional Astronomy and Celestial Mechanics; Peniscola, Spain, Oct.7.11. (to appear).

Taylor, D. B.: 1984, Astron. Astrophys., 141, 151-158.

Taylor, D. B., Sinclair, A.D., Message, P.J.: 1987, Astron. Astrophys., 181, 383-390.

Taylor, D. B.: 1992, Astron. Astrophys., 265, 825-832.

Woltjer, J., Jr.: 1928, Annalen van de Sterrewachte Leiden XV. 